\title{
Elastic Coupling Effects in Cooperative Transport by a Pair of Molecular Motors
}

\author{
Florian Berger, Corina Keller, Reinhard Lipowsky, and Stefan KlumpP \\ Max Planck Institute of Colloids and Interfaces, Science Park Golm, 14424 Potsdam, Germany \\ (Received 11 September 2012; accepted 5 November 2012; published online 27 November 2012)
}

\begin{abstract}
Engineered constructs coupling a defined number of molecular motors provide an opportunity to study the cooperative transport of cargoes. Theoretical descriptions for the dynamics of such complexes can help to understand experimental data or to quantitatively formulate expectations for such experiments and provide a general framework for such analysis. Here, we review and extend recent theoretical studies that focused on pairs of molecular motors to study effects of coupling between the motors. We derive explicit results for two elastically coupled kinesin-1 motors as a function of the coupling strength using both linear and nonlinear springs. In addition, we discuss the general dynamics of such motor pairs, which is governed by characteristic time scales for the spontaneous unbinding of motors and for the built-up of strain forces that are sufficiently large to affect the run length and/or the velocity of the motors. We show how the comparison of these time scales can be used to predict the distinct behavior of different motor species, the effects of coupling, and the impact of the single motor velocity on the observable dynamics of a motor pair.
\end{abstract}

Keywords-Molecular motors, Cooperative cargo transport, Elastic coupling, Multi-motor constructs, Stochastic modeling, Kinesin, Myosin V, Dynein.

\section{INTRODUCTION}

Force generation and active movements by cytoskeletal motors such as kinesins, dyneins, and myosins are crucial for long-range transport in cells and for the self-organization of their complex internal structure $^{31,47,55}$ They have also provided a blueprint for the design of engineered nanoscale transport systems ${ }^{26,27,40}$ and inspired the general theoretical study of nanoscale molecular machines. ${ }^{47}$ Our current understanding of how these molecular motors work has been advanced greatly by the use of single molecule technology such as optical tweezers,${ }^{58}$ which allowed

Address correspondence to Florian Berger, Max Planck Institute of Colloids and Interfaces, Science Park Golm, 14424 Potsdam, Germany. Electronic mail: florian.berger@mpikg.mpg.de for the quantitative characterization of the motor's behavior under the influence of external force. Together with theoretical descriptions of their kinetics, ${ }^{22,46}$ the data from single molecule experiments provide a detailed picture of the dynamics of individual motors.

In cells, however, motors often work in teams rather than as single molecules. This has been known for decades for the assemblies of millions of myosin motors in muscle, ${ }^{32,33}$ but more recently it has also been realized that cytoskeletal cargo transport relies on motor cooperation as well, although here the number of motors forming a team is typically small (often between 1 and 10). ${ }^{25}$ In the simplest case, a cargo may be pulled by several motors of the same type, for example several kinesin-1 motors. Moreover, cellular cargoes are often associated with motors belonging to different motor species, which further increases the complexity of cargo transport: Many cargoes move bidirectionally along microtubules due to the presence of both kinesin and dynein motors, which both walk along microtubules, but in opposite directions. ${ }^{24,62}$ In addition, cargoes may switch from microtubule-based transport to transport along actin filaments, if myosin motors are present as well. ${ }^{30}$ The cooperation of different motor species obviously extends the range of dynamical behavior that can be achieved with molecular motors. ${ }^{6}$ Beyond that, there are several advantages that teams of motors provide over individual motors. Most importantly they may be able to generate larger overall forces. ${ }^{60}$ In addition motor cooperation can lead to longer runs, ${ }^{3,10,17}$ in extreme cases up to millimeter. ${ }^{12}$ Motor cooperation may also result in an increased velocity in situations where the motors work against an opposing load force if multiple motors share the load. ${ }^{23}$ However, a recent study suggested that the load may not always be shared equally by the motors. ${ }^{34}$ Finally, transport by multiple motors provides additional opportunities for the regulation of transport as illustrated by the effects of microtubule-associated proteins. ${ }^{60,18}$

In recent years, it has therefore become more and more evident that to understand transport processes in 
cells, one has to move beyond single motors and study the cooperative action of several motors or motor assemblies and the mechanism by which these motors are coordinated. ${ }^{15,25,38}$ The first such studies used beads covered by several kinesin or dynein motors. ${ }^{3,49,60}$ While these experiments provided some insight into motor cooperation, they remained limited by the fact that the number of cooperating motors could only be controlled on average. An important improvement for studying motor cooperation was thus the design of synthetic assemblies of precisely defined numbers of motors. ${ }^{48,53,63}$ Since then a number of studies have investigated such defined motor complexes: The most extensive studies have been done for a system of two kinesin-1 motors coupled via a DNA scaffold, ${ }^{34,53}$ but other studies have also used two kinesins coupled via an antibody, ${ }^{63}$ two coupled myosin $\mathrm{V}$ motors,${ }^{48}$ and a myosin V motor coupled to a myosin VI motor. ${ }^{1}$ Motor cooperation has also been studied with microtubule gliding assays, in which motors immobilized on a surface pull a microtubule along that surface. ${ }^{8,45}$

In parallel to these experimental efforts, the dynamics of teams of cytoskeletal motors has also been tackled by theoretical and computational approaches. A general theoretical framework for studying motor teams has been proposed in 2005 by our group. ${ }^{38}$ This framework has been used to study unidirectional transport by one team of motors belonging to the same motor species ${ }^{3,38}$ and was later extended to bidirectional transport by two teams of motors. ${ }^{50-52}$ In addition, several groups have developed models that include detailed descriptions of the single motors involved and that address specific effects such as the stochasticity of stepping ${ }^{19,41,42}$ and the geometry of motor assemblies. ${ }^{13,20,21,40}$ As in the experimental studies listed above, the focus of recent theoretical work has been on assemblies of two motor molecules. In particular, several recent studies have addressed the model case of two coupled kinesin-1 motors. Somewhat surprisingly, these studies came to qualitatively different conclusions. One aspect that has been in the focus is the question whether there is "motor interference", i.e. whether motors impede their movements by exerting forces on each other due to their stochastic stepping. Experimentally, the interference issue was emphasized by the observation that unbinding of a motor in a twokinesin assembly is enhanced when both motors are bound to the microtubule. ${ }^{53}$ While some modeling studied have reproduced this effect, ${ }^{19,20,36}$ others have not or have in addition predicted a decrease of the velocity ${ }^{41,61,64}$ that has not been observed. This confusing (theoretical) situation has led us to re-examine the case of two coupled motors. ${ }^{7,37}$

In a recent study ${ }^{7}$ we have shown that such systems can exhibit four distinct transport regimes that depend on both the parameters of the motor molecule itself and on the elastic coupling between the motors. We have proposed simple time scale arguments that lead to an intuitive picture of the effects of elastic coupling between two motors. In the present article, we continue this line of thought. We use these time scale arguments and the theoretical description of motor pairs developed in Berger et al. ${ }^{7}$ to address the effects of various types of nonlinear elasticity and of different ways of varying the single motor velocity. In addition, we also provide explicit results for observable properties of an assembly of two kinesins.

The article is organized as follows: We first introduce the theoretical approach which is based on the general framework proposed in Klumpp and Lipowsky ${ }^{38}$ and the detailed model for two elastically coupled motors of Berger et al. ${ }^{7} \mathrm{We}$ also phrase the interference problem in terms of the general description of Klumpp and Lipowsky. ${ }^{38}$ Then we present explicit results for two coupled kinesin motors obtained with this approach, focusing on the dependence of the result on the coupling constant. Finally, the last part of the article deals with the time scale arguments. We discuss how they can explain the different transport regimes found in our previous study and how they predict different patterns of motor interference for different types motor assemblies, specifically of kinesin-1 motors and of myosin $\mathrm{V}$ motors. We then demonstrate the use of these time scale arguments by addressing the effects of nonlinear coupling between the motors, as well as the results of varying the single motor velocity. As a result of these theoretical considerations, we conclude that an experimental study of motor assemblies that differ only in the coupling between the two motors would be desirable.

\section{THEORETICAL DESCRIPTION OF PAIRS OF COUPLED MOLECULAR MOTORS}

\section{General Theoretical Framework}

Our starting point for a theoretical analysis is a general theoretical framework for transport by several molecular motors, ${ }^{38}$ which we use here in the special case of two identical motor molecules. Within this theoretical description, a motor can bind to the filament, walk along it, and unbind from it. The state of a cargo is characterized by the number of bound motors. For a cargo transported by a motor pair there are thus three states $C_{0}, C_{1}$, and $C_{2}$ in which the cargo is transported by 0,1 or 2 motors, respectively (Fig. 1). Since we are interested in the movement of the cargo on length scales that are large compared to the motor step size $l \simeq 8 \mathrm{~nm},{ }^{17}$ we characterize each cargo state with an (average) velocity $\left(v_{1}\right.$ and $\left.v_{2}\right)$. Transitions between the cargo states occur when a motor binds to 


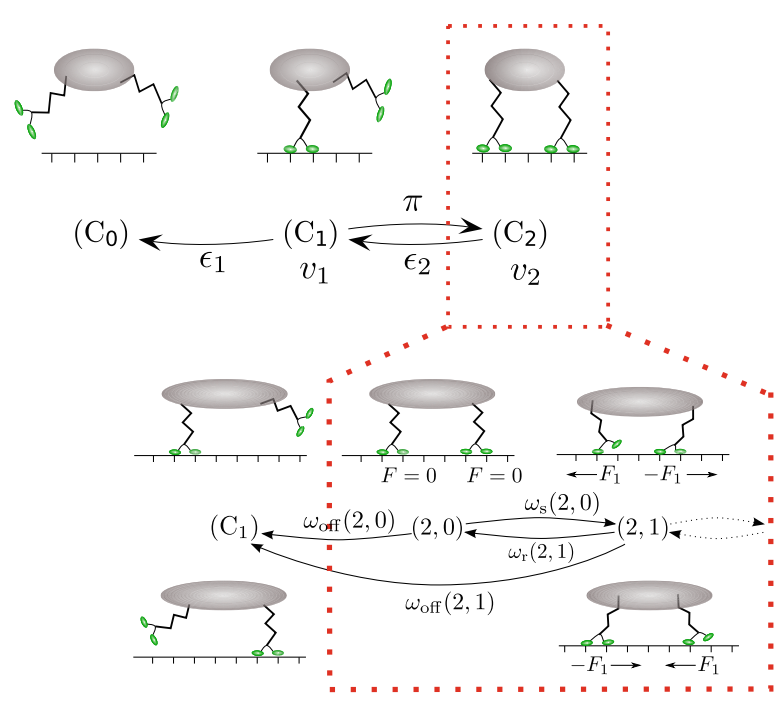

FIGURE 1. Theoretical description of two coupled molecular motors. The upper row shows the states of a cargo transported by two motors, where these states are defined by the number of motors linking the cargo particle to the filament: In state $C_{1}$, the cargo is pulled by one motor and in state $C_{2}$ by two motors. In addition, it can unbind from the filament into the absorbing state $C_{0}$. In state $C_{1}$ and state $C_{2}$ the velocity of the cargo is $v_{1}$ and $v_{2}$, respectively. The arrows indicate transitions between the states with their associated rates, the single motor unbinding rate $\epsilon_{1}$, the effective unbinding rate $\epsilon_{2}$ of one of the motors in a motor pair and the single motor binding rate $\pi$. The two lower rows display our detailed mechanistic model of two coupled motors that resolves state $C_{2}$ into microstates with different configurations of the motor pair characterized by the strain force between the two motors: In state $(2,0)$, the motors are bound with relaxed linkers such that there is no force between the motors. When the motors step either towards each other or away from each other, a strain force is generated between them, as, e.g., in state $(2,1)$; there are two motor configurations that correspond to this state. Transitions between the states correspond to stretching or relaxing of the elastic linkers due to stepping of a motor (with rates $\omega_{s}(2, i)$ and $\omega_{r}(2, i)$ respectively). Unbinding of a motor occurs with the rate $\omega_{\text {off }}(2,1)$. All these rates depend on the force and thus on the state $(2, i)$. The space of microstates in the lower row is used to calculate the effective quantities $\epsilon_{2}$ and $v_{2}$ that result from the interplay of stochastic stepping, strain force-generation, and unbinding.

or unbinds from the filament. An individual motor unbinds with rate $\epsilon_{1}$; this rate also determines the transition rate from state $C_{1}$ to state $C_{0}$, which occurs when the only bound motor unbinds. The transition from $C_{2}$ to $C_{1}$ is also based on unbinding of a motor, more precisely on the unbinding of one out of the two bound motors. Its rate, termed $\epsilon_{2}$ will depend on the single motor unbinding rate but is not identical to it. Finally, binding of the second motor (when one is bound) determines the transition from $C_{1}$ to $C_{2}$ and is described by a binding rate $\pi$. We assume that every run of a cargo starts with an unbound cargo first binding to a filament via one motor into state $C_{1}$.

The theoretical framework described so far allows one to calculate explicit expressions for a number of observable properties of the runs of cargoes pulled by several motors. ${ }^{38}$ For example, the mean run time, i.e. the average duration of a cargo run from initial binding to a filament to complete unbinding, is given by

$$
\left\langle\Delta t_{\mathrm{ca}}\right\rangle \equiv \frac{\pi+\epsilon_{2}}{\epsilon_{1} \epsilon_{2}}
$$

and the mean run length by

$$
\left\langle\Delta x_{\mathrm{ca}}\right\rangle \equiv \frac{\pi v_{2}+\epsilon_{2} v_{1}}{\epsilon_{1} \epsilon_{2}} .
$$

The average velocity $v_{\mathrm{ca}}$ is obtained from the ratio,

$$
v_{\mathrm{ca}} \equiv \frac{\left\langle\Delta x_{\mathrm{ca}}\right\rangle}{\left\langle\Delta t_{\mathrm{ca}}\right\rangle} .
$$

Furthermore, the distributions of these quantities can be also calculated analytically, see Klumpp and Lipowsky. ${ }^{38}$

The description given so far provides a general framework for the analysis of transport by several motors, but to make quantitative predictions, the transition rates need to be specified. This is relatively easy for state $C_{1}$, where the cargo is transported by only one motor. The parameters of this state, the unbinding rate $\epsilon_{1}$ and the velocity $v_{1}$, can thus be taken from measurements for individual motors. The binding rate $\pi$ is difficult to measure directly and depends in general on the geometry of the assembly and other typically poorly controlled factors. It has been determined indirectly (by fitting experimental data with a model) for kinesin-pulled membrane tubes, kinesindriven beads and two-kinesin complexes. For the first two systems a binding rate of $\simeq 5 / \mathrm{s}^{3,44}$ was found, while the latter resulted in smaller binding rates. ${ }^{53,63}$

The parameters $\epsilon_{2}$ and $v_{2}$ that characterize state $C_{2}$ are even less straightforward. In the case of non-interacting motors, in which the motors step independently of each other, we obtain $v_{2}=v_{1}$ and $\epsilon_{2}=2 \epsilon_{1}$, since the rate for an unbinding event when both motors are bound, is the sum of the single rates. ${ }^{38}$ Such non-interacting behavior is the extreme case of a very loose coupling between the motors. However, in general, the parameters $\epsilon_{2}$ and $v_{2}$ depend on the dynamical parameters of a single motor and on the interactions between the motors. In principle they can be determined experimentally by careful analysis of trajectories of two-motor complexes provided that the cargo states $C_{1}$ and $C_{2}$ can be identified. This approach has been taken in a series of recent studies by Diehl and collaborators, ${ }^{34,48,53}$ who distinguish microstates with one or two load-bearing motors, although these do not necessarily correspond to the states $C_{1}$ and $C_{2}$ with one or two bound motors, if the motors do not share the load. Likewise, in gliding assays, trajectories can be partitioned into segments with one or two motor pulling the microtubule based on the different swiveling behavior of the microtubule. ${ }^{45}$ 
In such approaches, the theoretical framework described above is not used in a predictive manner but rather as an analysis tool. For example, a key result of the studies of Diehl et al. was the observation of motor interference, enhanced unbinding of motors in a twokinesin complex when two motors are bound and, as a consequence relatively short run lengths. This result was obtained by comparing the experimentally determined rate $\epsilon_{2}$ for a 2-kinesin complex with what is expected if the two motors unbind independently, without any interactions. It is however worth mentioning that earlier experiments on kinesin-driven beads were consistent with the long run lengths expected for non-interacting motors ${ }^{3}$ or showed run lengths even longer than expected. ${ }^{60}$

In order to make quantitative predictions using the theoretical framework outlined above, one either needs to specify the remaining parameters (i.e. those that are not directly given by single motor observations such as $\epsilon_{2}$ and $v_{2}$ ) based on plausible assumptions or determine them from a more microscopic model. To tackle this task several groups (including ours) have developed a variety of detailed models for coupled motors. These approaches typically take one of two roads: They either start by splitting the state with two bound motors, $C_{2}$ into several substates, ${ }^{7,14,19,20,37,57,61}$ in which case some results can still be obtained analytically, or use detailed computational models that particularly emphasize on the geometry of the motor-complex or the arrangement of motors on a bead. ${ }^{13,39,41}$ In reference, ${ }^{37}$ a detailed chemomechanical network for two-motor complexes is used to generate trajectories and to show that the parameters $\epsilon_{2}$ and $v_{2}$ can be deduced in a unique manner from the statistical properties of the trajectories. As mentioned already in the introduction, there exist considerable differences between the predictions obtained from different models with respect to interference effects.

Below we will follow the first type of approach using the general model described in Berger et al. ${ }^{7}$ to obtain specific results for coupled kinesin-1 motors and to discuss effects of different types and strengths of coupling (we note that we have also pursued a complementary approach to coupled motors, ${ }^{37}$ which starts from a detailed description of the chemomechanical dynamics of a single motor). We will specifically be interested in the build-up dynamics of force between the motors to study the conditions under which elastic coupling between the motors leads to interference effects such as enhanced unbinding $\left(\epsilon_{2}>2 \epsilon_{1}\right)$, as observed for kinesin pairs, ${ }^{53}$ or a reduced velocity $\left(v_{2}<v_{1}\right)$, as observed for pairs of myosin V motors. ${ }^{48}$ This type of motor interference depends on the single motor dynamics, in particular on the reaction of the motor to a force. Knowing the force-dependent dynamics of a single motor from optical trapping experiments, it is possible to predict the dynamics of two elastically coupled motors. Following this line of thought, we next introduce a theoretical description for two elastically coupled molecular motors that provides a detailed description of state $C_{2}$, in which both motors actively pull the cargo. This theory is based on a single motor description that captures all relevant characteristics as known from single molecule experiments.

\section{Theoretical Description of Coupled Motors}

Our detailed description of coupled molecular motors that interact by exerting forces on each other is based on the force-dependent dynamics of individual motors that has been characterized extensively via single molecule experiments. Our description of single motors is summarized in the "Appendix". This description is predicated on experimental observations for kinesin-1, but can be adjusted to other motors as well. It describes the following dynamic processes: binding of the motor to the filament, unbinding, and forward stepping (in a simple extension of the model, backward steps can also be taken into account ${ }^{7}$ ). These three processes are characterized by the corresponding rates, which, in principle, could all be force-dependent. However, as in several previous studies ${ }^{38,50}$ (but different from others ${ }^{19,20}$ ), we take the binding rate $\pi$ to be independent of force. A justification for this simplification is given in the "Appendix". As a consequence, we are left with two force-dependent processes, unbinding and stepping. The two force-dependencies are characterized by two characteristic force scales, the detachment force $F_{d}$, which governs the increase of the unbinding rate as a function of force, and the stall force $F_{s}$ at which the velocity of the motor vanishes. These two characteristic force scales are crucial for understanding the dynamics of a motor pair.

With this description of single motors at hand, we come back to the description of a cargo transported by two motors. Our task is to deduce the parameters $\epsilon_{2}$ and $v_{2}$ from a microscopic model. ${ }^{7}$ This model provides a more detailed description of the cargo state $C_{2}$. In this state both motors are bound to the filament and pull the cargo. The motors are coupled via their elastic linkers to the common cargo, and the strain force developed in this elastic element is a function of its extension. Because of the discrete stepping of the motors the extension is a discrete quantity and, thus, the force between the motors can only have the discrete values

$$
F_{i} \equiv F\left(x_{i}\right) .
$$

We now describe the state of the cargo by the discrete extension of the elastic elements, which defines a series of substates $(2,0) \ldots(2, N)$ of state $C_{2}$, see Fig. 1. Every state $(2, i)$ is characterized by a force $F_{i}$ acting on the two motors or a corresponding extension 
$x_{i}$ of the elastic element. In state $(2,0)$ the linkers are relaxed. If one of the motors steps, the absolute extension of both linkers increases by the motor step size $l$ and the cargo is in state $(2,1)$. For identical motors each linker is stretched by $l / 2$. This stretching induces a strain force between the motors. One motor feels the force $F_{1}=F(l / 2)$ and the other motor feels the opposing force $-F_{1}=-F(l / 2)$. To calculate the forces $F_{i}$ explicitly, the nature of the elastic element needs to be specified. In the simplest case, we consider the motor stalks as linear elastic springs with stiffness $\kappa$. Then $F_{i}$ is obtained from the force-extension relation of two such linear springs in series, $F_{i}=\kappa l i / 2$. More complex types of coupling will be considered below.

Transitions between the states $(2,0) \ldots(2, N)$ occur whenever a motor steps. We denote the transition rates for extension of the linkers by $\omega_{s}$ and for relaxation by $\omega_{r}$. These transition rates are connected to the stepping rates of the single motors under force and are obtained by summing up the rates of all steps that result in extension or relaxation, respectively, ${ }^{7}$ which leads to

$$
\omega_{\mathrm{s}}(2, i)=\alpha\left(F_{i}\right)=\mathcal{V}\left(F_{i}\right) / l
$$

and

$$
\omega_{\mathrm{r}}(2, i)=\alpha\left(-F_{i}\right)=\mathcal{V}\left(-F_{i}\right) / l
$$

for all states $(2, i)$ with $i>0$ and

$$
\omega_{\mathrm{s}}(2,0)=2 \alpha(0)=2 \mathcal{V}(0) / l
$$

for state $(2,0)$. In these expressions $\mathcal{V}(F)$ denotes the forcevelocity relation of a single motor. Next, we have to consider the unbinding of the motors. Unbinding of one motor is described by transitions from every state $(2,0) \ldots(2, N)$ to the state $C_{1}$ in which the cargo is transported by a single motor. The corresponding transitions rates are given by the force-dependent unbinding rates,

$$
\omega_{\text {off }}(2, i)=\epsilon_{1}\left(F_{i}\right)+\epsilon_{1}\left(-F_{i}\right) .
$$

With these definitions, we have expressed all rates in terms of the single motor unbinding rate $\epsilon_{1}(F)$ and force-velocity relation $\mathcal{V}(F)$ (which could be taken directly from experimental data, for the description we use here, see the "Appendix"). To calculate the two quantities $\epsilon_{2}$ and $v_{2}$, which are determined by the dynamics when both motors are bound, we treat the state $C_{1}$ as an absorbing state. The unbinding rate $\epsilon_{2}$ is then calculated as the inverse of the mean first passage time to absorption and $v_{2}$ as the average stepping rate times the displacement, as shown in the "Appendix".

\section{TWO COUPLED KINESIN MOTORS}

The theoretical framework developed so far allows us to calculate the two parameters $\epsilon_{2}$ and $v_{2}$ of the two-motor bound state $C_{2}$. In Fig. 2 we show these parameters as a function of the spring constant $\kappa$ for the kinesin parameters of Table 1. As expected, as the coupling strength is increased, the velocity is reduced, while the unbinding rate increases, indicating that the motors exert force on each other that interfere with their function. It is however worth noting that for spring constants smaller than $0.3 \mathrm{pN} / \mathrm{nm}$, the velocity is only reduced by $25 \%$ whereas the unbinding rate is increased by $100 \%$ compared to the non-interacting case $(\kappa=0 \mathrm{pN} / \mathrm{nm})$. Hence, we draw the conclusion that kinesin motors interfere in such a way that they pull each other off the filament, while maintaining an almost constant velocity. Below this effect will be explained by the comparison of two time scales characterizing the built-up of force sufficient to force motor unbinding and to cause significant slow-down of their stepping, respectively.

The two parameters $\epsilon_{2}$ and $v_{2}$ calculated so far characterize the state of the cargo where it is bound to a filament through both motors. They can be measured directly if trajectories can reliably be partitioned into sequences of 1-motor runs and 2-motor runs, i.e. into segments where the cargo is bound to the filament through one or two motors, respectively, see also Keller et al. ${ }^{37}$ The overall motility of such cargoes are characterized by the average velocity and the overall run length, which may also be determined without the need to resolve such partitioning of the trajectories. Within the model, these quantities can be calculated via Eqs. 1 and 2. They are plotted in Figs. 2(c) and 2(d), again as functions of the spring constant $\kappa$. Both quantities depend also on the binding rate $\pi$, in contrast to the parameters $\epsilon_{2}$ and $v_{2}$, which do not. We therefore show data for two different values of the binding rate. The behavior of the two global transport characteristics reflects the dependence of the parameters of state $C_{2}$ : The run length exhibits a strong decrease as the coupling strength is increased, reflecting the increase in the unbinding rate $\epsilon_{2}$. We note, however, that this reduction is a reduction compared to the case of non-interacting motors and not a reduction compared to transport by one motor. In fact, the run length of a two-motor assembly is always found to be longer than that of a single motor. Only in the limit of very strong coupling, we recover the run length of a single motor, as in that limit the second motors unbinds immediately after binding.

The average velocity of the cargo is reduced much less than the run length, as expected based on the weak effect of the coupling on $v_{2}$. In fact for sufficiently strong coupling, we find that the average velocity increases again. This observation can be explained by two counteracting effects. On the one hand, the velocity in state $C_{2}$, where both motors are bound, 
(a)

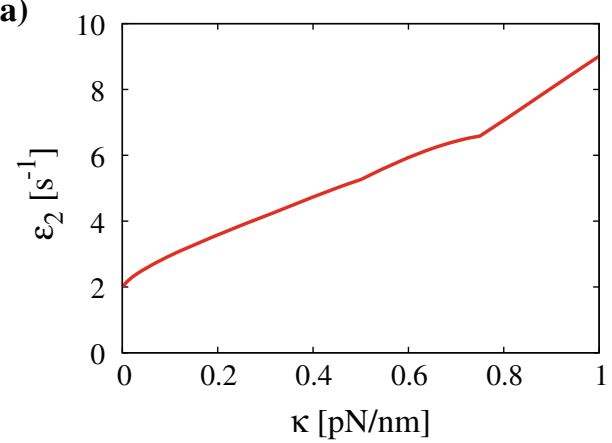

(c)

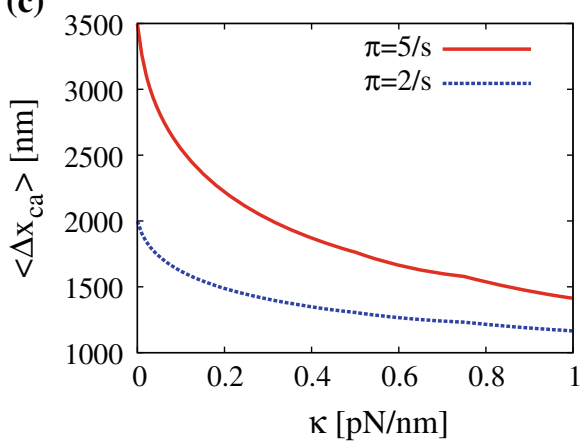

(b)

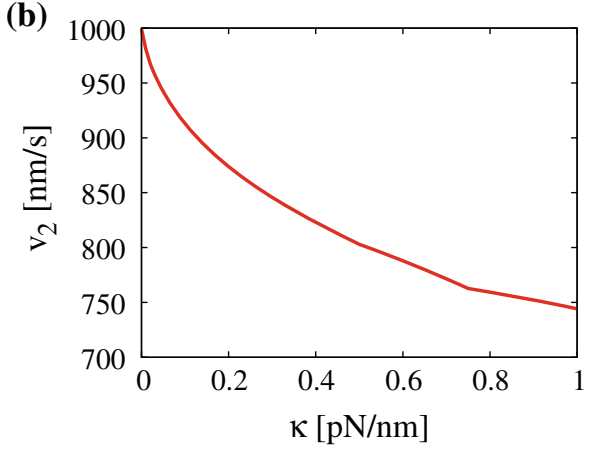

(d)

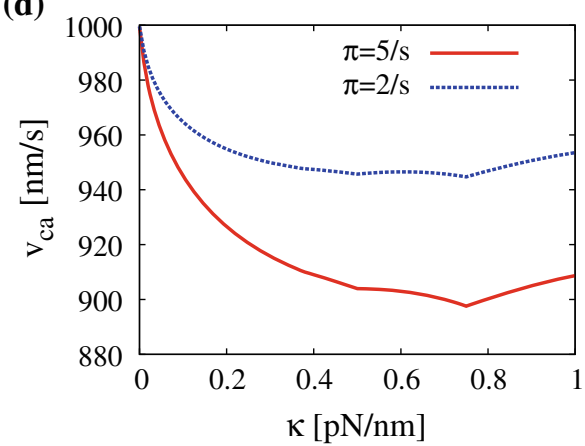

FIGURE 2. Characteristics of the dynamics for an elastically coupled kinesin pair and dependence on the coupling strength: (a) effective unbinding rate $\epsilon_{2}$ as a function of the spring constant $\kappa$. For stronger coupling the unbinding rate increases; the motor pull each other from the filament. For weak coupling the unbinding rate attains the value $\epsilon_{2}=2 \epsilon_{1}$, as expected for non interacting motors; (b) average velocity $v_{2}$ of a cargo simultaneously transported by two both motors. For strong coupling, the velocity decreases. For weak coupling the cargo is transported with the single motor velocity; (c) overall cargo run length $\left\langle\Delta x_{\mathrm{ca}}\right\rangle$ as in Eq. (2) and (d) cargo velocity $v_{\mathrm{ca}}$ as in Eq. (3) obtained with the rates of (a) and (b) for two different binding rates $\pi$. For a larger binding rate, the cargo is more often transported by both motors and therefore, the motor-motor interference effects are more pronounced in the dynamics of the overall cargo. In all plots, the single motor parameters are those given in Table 1.

TABLE 1. Parameters used for the single kinesin description.

\begin{tabular}{lll}
\hline Parameter & Symbol & Value \\
\hline Stall force & $F_{\mathrm{s}}$ & $6 \mathrm{pN}^{56}$ \\
Detachment force & $F_{\mathrm{d}}$ & $3 \mathrm{pN}^{56}$ \\
Force-free velocity & $V_{1}$ & $1 \mu \mathrm{m} / \mathrm{s}^{56}$ \\
Force-free unbinding rate & $\epsilon_{0}$ & $1 \mathrm{~s}^{-156}$ \\
Binding rate & $\pi$ & $5 \mathrm{~s}^{-13,44}$ \\
step size & $I$ & $8 \mathrm{~nm}^{17}$ \\
\hline
\end{tabular}

decreases (Fig. 2b), but on the other hand, as $\epsilon_{2}$ increases the probability of being in that state decreases as well. The overall effect on the average velocity is thus even smaller than the effect on $v_{2}$, with approximately 5 and $10 \%$ maximal reduction in the two cases we show. For both quantities, the impact of coupling is more pronounced (i.e. the fold-change compared to the non-interacting case is larger) for larger binding rate $\pi$. This is, of course, due to the increased fraction of time the cargo spends in state $C_{2}$ with both motors bound, as coupling effects only play a role in this state.

\section{USING COMPARISONS OF TIME SCALES TO UNDERSTAND COUPLED MOTORS}

The complex behavior of two coupled motors with several distinct transport regimes can be understood in a more intuitive way by comparing different time scales associated with the generation of strain forces between the motors. In this section, we briefly review the time scale argument introduced in our earlier work ${ }^{7}$ and then use these arguments to explain the effects of nonlinear coupling and of a modulation of the single motor velocity. We wish to emphasize that the salient time scales can be calculated exactly within the theoretical framework developed in the previous section, but that an alternative use of such arguments is to make qualitative or semi-quantitative predictions based on a intuitive picture of how the built-up of strain may be accelerated or delayed by changing various parameters of the system or the type of coupling between the motors.

\section{Time Scales for Strain Force Generation}

The dynamics of cooperative motor transport is governed by the interplay of three processes: the 
stepping of the motors, the unbinding of the motors and the build-up of strain forces between them. Thus, strongly coupled and/or fast motors can quickly build up a large strain force that pulls one of the motors from the filament, while weakly coupled and/or slow motors may unbind spontaneously before reaching such a large force.

In order to systematically study which process dominates and how that depends on the single motor properties and on the coupling, we compare three different time scales: the time scale for spontaneous unbinding of a motor and the two times it takes to build up motormotor forces that are comparable to the detachment and stall force, respectively. An illustration of these time scales is shown in Fig. 3. The time scale for spontaneous unbinding $t_{u}$ is the average time that a non-interacting motor pair remains bound to the filament via both motors, i.e. the average time until one of the two motors unbinds. The time scale $t_{F d}$ for force induced unbinding, is the time it takes to built up a strain force between the motors that is comparable to the detachment force. The time scale $t_{F s}$ for force-induced stalling is the time that is required to generate a strain force between the motors that is comparable to the stall force. These time scales can be calculated from detailed mean first passage time calculations, see Berger et $\mathrm{al}^{7}{ }^{7}$ The comparison of these time scales allows us to develop an intuitive understanding of the cooperative transport of two elastically coupled motors as described in the following.

\section{Distinct Transport Regimes for Different Motor Species}

For two elastically coupled kinesin motors, the unbinding rate increases dramatically as a function of the coupling strength, while the velocity is only weakly affected, see Figs. 2(a) and 2(b). Thus, unbinding is the dominant process in such a system. This conclusion also follows from the comparison of the time scales. For kinesin-1, the detachment force $F_{\mathrm{d}}$ is smaller than the stall force $F_{\mathrm{s}}$, and the strain force built up between the motors will first reach the detachment force and hence $t_{F d}<t_{F s}$. Unless the coupling is very weak, these times are also shorter than the time for spontaneous unbinding $t_{u}$ and thus force-induced unbinding is the dominant process.

In our previous study, we have determined the parameters $\epsilon_{2}$ and $v_{2}$ systematically as a function of the motor parameters and the coupling strength and identified four distinct regimes. ${ }^{7}$ The parameter that could be varied experimentally for a given motor pair is the coupling strength. Within the theory, a variation of the spring constant corresponds to a path through the different transport regimes, which however need not cross all four regimes. For example, kinesin-1 motors can attain the "enhanced unbinding regime" described so far, a weak coupling regime and a strong coupling regime. In the weak coupling regime, for small $\kappa$, there is almost no effect on the unbinding rate and on the velocity, whereas the strong coupling regime, for large $\kappa$, is characterized by an enhanced unbinding rate and a reduced velocity. Each regime corresponds to an ordered sequence of the three time scales, under the constraint that $t_{F d}<t_{F s}$ : Weak coupling is obtained for $t_{u}<t_{F d}<t_{F s}$, enhanced unbinding for $t_{F d}<t_{u}<t_{F s}$, and strong coupling for $t_{F d}<t_{F s}<t_{u}$. The observations by the Diehl lab correspond to the enhanced unbinding regime characterized by an enhanced unbinding rate with only a small
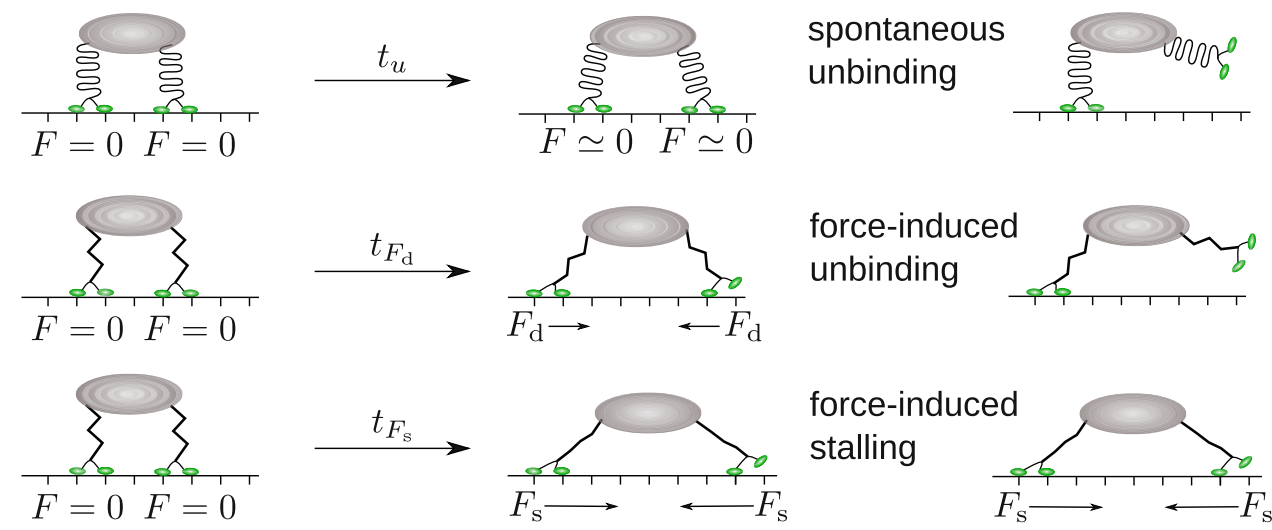

time

FIGURE 3. Different time scales associated with the competing processes of spontaneous unbinding, force-induced unbinding and force-induced stalling: The time scale $t_{u}$ for spontaneous unbinding is the time, after which one of two weakly coupled motor unbinds (upper row). The time scale $t_{F d}$ for force induced unbinding is the time two coupled motors need to build-up a strain force comparable to the detachment force (middle row). The time scale $t_{F s}$ for force-induced stalling is the time two elastically coupled motors need to build-up a strain force that is of the order of the stall force (lower row). 
effect on the velocity. ${ }^{53}$ As a side remark, we also note that the comparison of time scales provides a natural explanation why interference is always obtained in models for coupled kinesins that assume that both motors remain bound all the time, ${ }^{14,61}$ as this case corresponds to the limit of infinite unbinding time $t_{u}$, which is part of the strong coupling regime.

Pairs of other types of motors may not attain the same transport regimes as kinesin. ${ }^{7}$ For example, for processive myosin motors, there is evidence that the detachment force is larger than the stall force. ${ }^{1}$ Considering this fact, our theory predicts that $t_{F s}<t_{F d}$ and, thus, that the dominant effect of motor-motor coupling should be a reduction of the velocity or stalling of the motors. The three different ordered sequences of the time scales now correspond to the weak and strong coupling regimes as above and to an intermediate "reduced velocity" regime. As a consequence, a cargo simultaneously transported by two processive myosin motors should have a reduced velocity without a substantially enhanced unbinding rate. $^{7}$ In fact, this has been reported very recently for coupled myosin $\mathrm{V}$ motors. ${ }^{48}$

\section{Different Coupling: Nonlinear Springs, Cable-like Springs}

Above and in our previous study, we have described the elastic coupling between the motors as a linear spring. In general, however, biopolymers may have nonlinear force-extension relations. Such non linear force-extension relations can easily be incorporated in our theoretical description via Eq. (4). In fact, as the model in Fig. 1 specifies the states of the 2-motor complex by the force acting between them, nonlinear coupling only modifies the values of the force associated with the different states and does not increase the complexity of the calculations. In the following, we consider several nonlinear spring models that have been used for kinesin motors. These models are based either on considerations from polymer physics to the kinesin stalk and tail domains or on experimental data for force-extension relations.

As a first example, we consider two nonlinear forceextension relations that have been reported for kinesin (plotted in Fig. 4a), based on an analysis of the fluctuations of the bead position in optical trapping assays. $^{2,19}$ In both cases, the force-extension relation increases slowly for small extensions, but fast for large extensions, corresponding to a soft spring that stiffens under force. For comparison, we also show a case with a linear spring, again using a stiffness reported for kinesin, $\kappa \simeq 0.3 \mathrm{pN} / \mathrm{nm} .{ }^{16}$ For all three cases, we calculated the unbinding rate $\epsilon_{2}$ and the velocity $v_{2}$ for the cargo state where both motors are bound (Figs. $4 \mathrm{~b}$ and $4 c)$. The overall results are similar in all three cases, but the interference effects are weaker for the two nonlinear springs: the unbinding rate is smaller and the velocity is larger than for the linear spring. This observation implies that the smaller stiffness of the nonlinear springs (for small extensions) is dominant, so the motors coupled via the nonlinear springs need more time to develop substantial strain forces. As thus strain is built up more slowly, the interference effects are less pronounced.

As a second example, we consider cable-like models for the coupling. Cable models have been used in several computational studies of cooperative motor transport. ${ }^{39,41,42}$ These studies have typically not seen strong interference effects. Cable models provide a simple description of the elasticity of a polymeric chain. In these models, the force-extension relation is split into two regions corresponding to compression and stretching of the polymeric linker. Under compression, i.e. for chain extensions smaller than its rest length $l_{0}$, no strain forces are generated, while strain is (a)

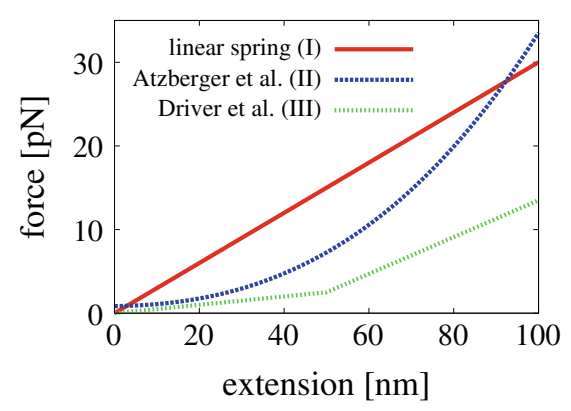

(b)

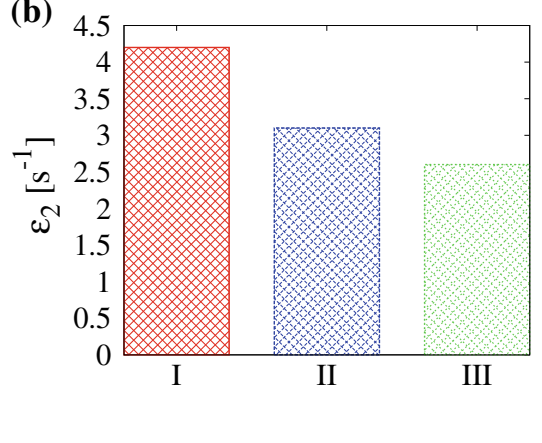

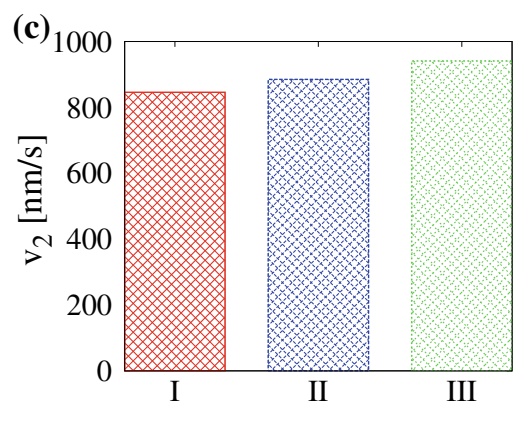

FIGURE 4. Nonlinear coupling of a motor pair: (a) Three force-extension relation for the kinesin linker as reported in different studies: (I) a linear spring (red) with stiffness $\kappa \simeq 0.3 \mathrm{pN} / \mathrm{nm},{ }^{16}$ (II) a polynomial nonlinear force-extension relation (blue) deduced by Atzberger and Peskin from experimental data. ${ }^{2}$ (III) A piecewise-linear force-extension relation (green) as suggested by Driver et al: ${ }^{19}$ (b) the unbinding rate $\epsilon_{2}$ and (c) the velocity $v_{2}$ for these three different linker models. For the nonlinear couplings the softspring behavior for small extensions dominates, resulting in smaller interference effects than for the stiffer linear spring. 
generated by stretching. In the stretching mode, i.e. for extensions larger than the rest length $l_{0}$, a linear forceextension relation applies. In the case of two coupled motors, two cables corresponding to the stalk of each motor are connected, which results in an effective rest length of $2 l_{0}$.

It is possible to implement a cable model within the framework used above, but because of the different behavior under compression and stretching this is more complicated than for the nonlinear springs considered so far and requires a few additional technical steps, which we describe in the "Appendix". It is however very simple to use the time scale arguments to obtain a semi-qualitative understanding of this case. Let us thus use these arguments to discuss what we should expect for a cable-like elastic element. When both motors start working together in the compression mode, where the linkers are relaxed, no forces are built up between the motors until their linkers are stretched to a distance larger than $2 l_{0}$. Thus the motors need a certain time to leave the compression mode. Likewise, motors starting with their linker at its rest length have a chance to enter the compression mode rather than the stretching mode. Both dynamic scenarios result in an offset time, given by the time it takes until the motors start to interact via their linkers. This offset time contributes to the times $t_{F d}$ and $t_{F s}$ required to generate relevant strain forces. For this reason, we expect, that in models using cable-like linkers, $t_{u}<t_{F d}$ and $t_{u}<t_{F s}$, and thus the interference is rather small or even vanishing as has been seen in previous studies using cable models. ${ }^{39,41,42}$

To test this argument, we have calculated the time scales exactly within the theoretical framework of the previous section, our calculation is based on an extended state space that accounts for cable-like linkers by allowing for additional states corresponding to the compression mode (described in the "Appendix"). Results of this detailed calculation are shown in Fig. 5. In Fig. 5a, we plot the time $t_{F d}$ for strain generation relevant for unbinding as a function of the rest length $l_{0}$ and compare it to the spontaneous unbinding time $t_{u}$. The time $t_{F d}$ increases with the rest length $l_{0}$. Already for a rest length of $l_{0}>30 \mathrm{~nm}$, we have $t_{F d}>t_{u}=1 / 2 \epsilon_{1} \simeq 0.5 \mathrm{~s}$ and the system is dominated by spontaneous unbinding as expected from the qualitative argument. In Figs. $5 \mathrm{~b}$ and $5 \mathrm{c}$, we show the corresponding results for the unbinding rate $\epsilon_{2}$ and the velocity $v_{2}$. Only for very short rest lengths, these parameters show effects of motor-motor interaction and deviate from the values expected for non-interacting motors $\left(v_{2}=v_{1}\right.$ and $\epsilon_{2}=2 \epsilon_{1}$, which are indicated by the blue lines). For large rest length $l_{0}$, interference effects in both the unbinding rate and the velocity are hardly visible, in agreement with the picture that the motors unbind spontaneously before generating substantial forces.

In summary, the motor linkers that act as cables decrease the elastic coupling strength and thus the interference effects. This conclusion agrees with previous studies based on cable models. ${ }^{39,41,42}$

\section{Control of Travel Distance Through the Velocity}

Another quantity that affects the time scales for the built-up of strain force is the value of the (single motor) velocity: As the strain is generated through motor stepping, force is built up more rapidly if motors step faster. As a consequence, interference effects should be enhanced by speeding up the motors and be reduced by slowing them down. Such variation of the velocity can, for example, be achieved by changing the concentration of ATP, which allows one to vary the velocity over two orders of magnitude..$^{54,56}$
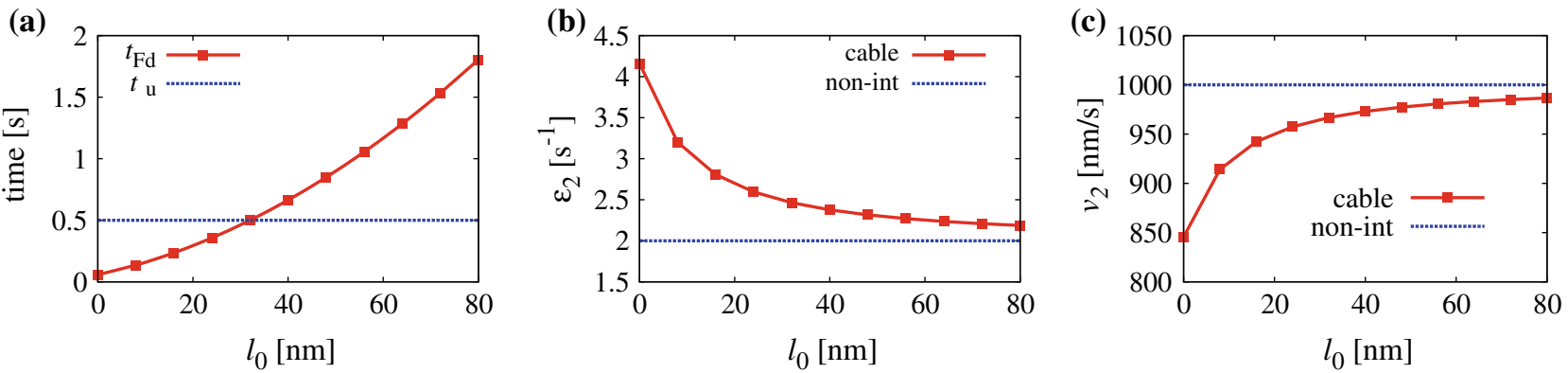

FIGURE 5. Cable-like coupling of the two motors: (a) Time scale $t_{F d}$ and $t_{u}$ as a function of the rest length $I_{0}$ of a cable-like linker (which exhibits a linear elastic response to stretching but none to compression). For large rest length, the time $t_{F d}$ to generate forces comparable to the detachment force is longer than the time $t_{u}$ for spontaneous unbinding. Thus, the system mainly unbinds spontaneously and interference effects are rather small; (b) unbinding rate $\epsilon_{2}$ and (c) velocity $v_{2}$ of two active kinesins with cablelike linkers as a function of the rest length $I_{0}$ of the cable. For $I_{0}$ approaching the linear extension of kinesin, which is about $80 \mathrm{~nm}$, the interference effect of the binding time and the velocity is strongly reduced. The blue lines indicate the value for non-interacting motors. 
The dependence of the dynamic parameters on the ATP concentration is, however, complex, as a variation of the ATP concentration modulates the chemomechanical cycle of a motor ${ }^{37}$ and may also affect other parameters in addition to the velocity. In particular, the ATP concentration may affect the (single motor) unbinding rate. In the case of kinesin-1, whether this is the case or not depends on the concentrations of ADP and inorganic phosphate in the medium. A detailed study of the effects of the ATP concentration will therefore require a description of the chemomechanical cycles of the motor. ${ }^{37}$ Here we consider two idealized limiting cases, for which the velocity $v_{1}$ is varied while either the unbinding rate $\epsilon_{1}$ or the ratio between the velocity and the unbinding rate $\left(v_{1} / \epsilon_{1}\right)$ remains constant. These two modes of modulating the velocity lead to rather different results.

We first consider the case, where the unbinding rate is considered as independent of the velocity and is thus kept constant. In this case, the time to build up strain between the motors is increased by slowing down the motors, while the time for spontaneous unbinding is unchanged. For slow motors, the latter time scale can be shorter than the time for building up a substantial force between the motors. Therefore, slow motors should be weakly coupled and behave as independent motors with $v_{2} \approx v_{1}$ and $\epsilon_{2} \approx 2 \epsilon_{1}$.

In Fig. 6, we plot the two parameters characterizing state $C_{2}, \epsilon_{2}$ and $v_{2}$ as well as the cargo run length as a function of the single motor velocity $v_{1}$. As expected from the time scale argument, the unbinding rate $\epsilon_{2}$ increases considerable as the single motor velocity is increased, indicating that force between the motors is built up more rapidly, see Fig. 6a. By contrast, the velocity exhibits an (almost) linear increase with $v_{1}$ (note that in Fig. 6b we plot the ratio $v_{2} / v_{1}$ which is almost constant). The linear dependence reflects the change in the prefactor of all rates related to stepping of a motor. Interference effects on the velocity are very weak, so only for strong coupling some nonlinear effects can be seen (the deviations from constant ratios in Fig. 6b) that reflect the slower built-up of strain force for small $v_{1}$. Likewise, for the run length of the cargo the dominant effect is the linear impact of the velocity, but there again are coupling-dependent nonlinear corrections, see Fig. 6c.

In the argument so far, we have assumed that the velocity is varied, while the unbinding rate remains unchanged. However, several experimental studies have shown that the run length of a single kinesin is (a)

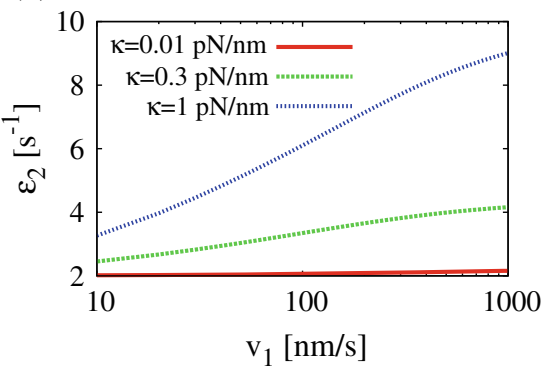

(d)

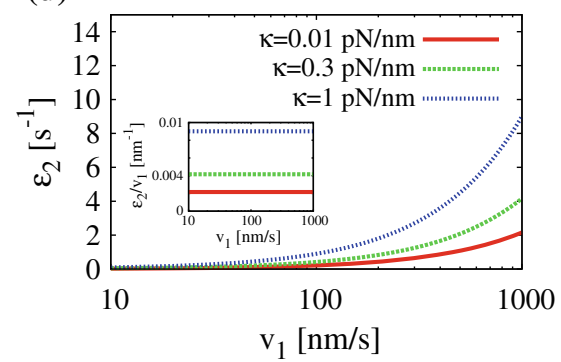

(b)

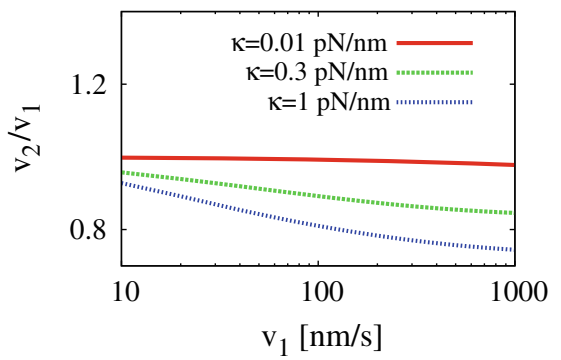

(e)

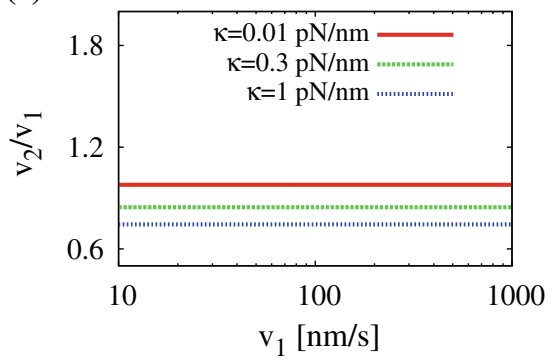

(c)

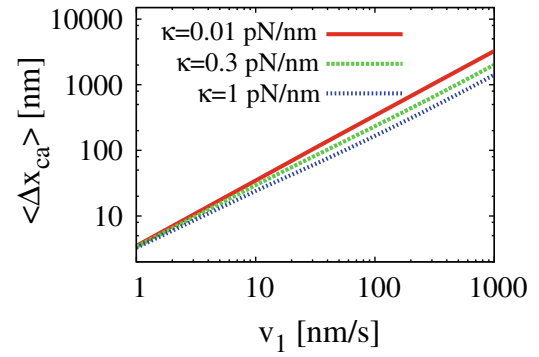

(f)

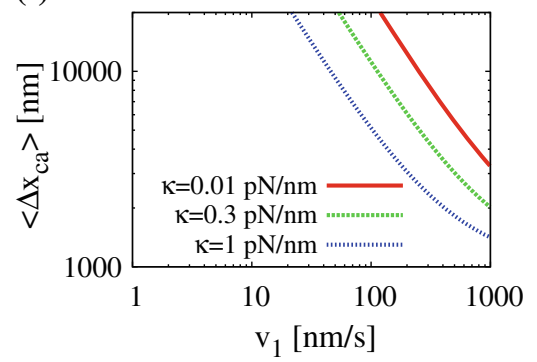

FIGURE 6. Effects of the single motor velocity on the dynamics of a motor pair: (a, d) Effective unbinding rate $\epsilon_{2} ;(\mathbf{b}, \mathbf{e})$ velocity $v_{2}$ and (c,f) overall cargo run length $\left\langle\Delta x_{\mathrm{ca}}\right\rangle$ for different spring constants $\boldsymbol{\kappa}$ as functions of the single motor velocity $v_{1}$. In plots (a)-(c), we vary $\boldsymbol{v}_{\mathbf{1}}$ while keeping the single motor unbinding rate $\epsilon_{1}$ constant, whereas in (d)-(f) we keep the ratio of the unbinding rate $\epsilon_{1}$ and $v_{1}$, i.e. the single Úmotor run length constant. (a) For constant $\epsilon_{1}$, the effective unbinding rate $\epsilon_{2}$ increases with the single motor velocity $v_{1}$, as strain between the motors is built up more rapidly; (b) the velocity $v_{2}$ is also affected and its ratio to $v_{1}$ decreases as a function of $v_{1}$; the effect on $v_{2}$ is relatively small, therefore we plot the ratio $v_{2} / v_{1}$; (c) the cargo run length increases as a function of the single motor velocity. Changing the single motor unbinding rate $\epsilon_{1}$ together with the single motor velocity $v_{1}$ leads to a different behavior; (d) the unbinding rate $\epsilon_{2}$ also increases with the single motor velocity $\boldsymbol{v}_{1}$, but this is only a scaling effect, the ratio $\epsilon_{2} / \boldsymbol{v}_{1}$ is constant (see the inset); (e) likewise, the two motor velocity $v_{2}$ also scales linearly with $v_{1}$; (f) as a result the overall cargo run length decreases as a function of the single motor velocity $v_{1}$, contrary to (c). 
independent of the ATP concentration over a rather wide range of ATP concentrations, ${ }^{59,63}$ contrary to what one would expect if the ATP concentration only affected the velocity. This observation indicates that a variation of the velocity via the ATP concentration also leads to a variation of the single motor unbinding rate $\epsilon_{1}$. It can be explained using models for the chemomechanical cycles of kinesin, ${ }^{46}$ which also show that this type of dependence is only expected for low concentrations of ADP and inorganic phosphate. This dependence can be interpreted as an effect of a constant (or approximately constant) unbinding probability per step rather than a constant unbinding rate, i.e. a constant unbinding probability per time. This feature has some interesting consequences for coupled motors that have been addressed experimentally in a recent study for two kinesins coupled via an antibody ${ }^{63}$ We note however that the run length becomes dependent on the ATP concentration if the ATP concentration is sufficiently low; ${ }^{56}$ here, what concentration is sufficiently low depends on the concentration of inorganic phosphate in the buffer. ${ }^{46}$

To discuss this issue within our theoretical framework, we consider a second mode of varying the single motor velocity. We express the single motor unbinding rate $\epsilon_{1}$ in terms of the single motor velocity $v_{1}$ and the run length $\langle\Delta x\rangle$ which leads to the relation $\epsilon_{1}=\frac{v_{1}}{\langle\Delta x\rangle}$. Inserting this relation into equation Eq. (2), and taking into account possible interference between the motors (which implied that the unbinding rate $\epsilon_{2}=\epsilon_{2}\left(v_{1}\right)$ and the velocity $v_{2}=v_{2}\left(v_{1}\right)$ are functions of the single motor velocity), we obtain the cargo run length as

$$
\left\langle\Delta x_{\mathrm{ca}}\right\rangle\left(v_{1}\right)=\langle\Delta x\rangle\left[1+\frac{\pi \cdot v_{2}\left(v_{1}\right)}{v_{1} \cdot \epsilon_{2}\left(v_{1}\right)}\right] .
$$

In the case of a velocity dependent unbinding rate, the rates of all dynamic processes within state $C_{2}$ are varied in parallel (all stepping rates are proportional to $v_{1}$ and all unbinding rates to $\epsilon_{1}$, which is also proportional to $v_{1}$ ). As a consequence, all time scales characteristic for state $C_{2}$ are equally affected, corresponding to an overall rescaling of the time unit. Thus, in this scenario, interference effects are not increased by increasing the velocity $v_{1}$; instead the presence or absence of interference (and its impact on transport) are independent of the values of $v_{1}$. As a consequence, both the unbinding rate $\epsilon_{2}$ and the velocity $v_{2}$ are linear functions of the single motor velocity $v_{1}$ (indicated by their constant ratios to $v_{1}$ ), simply reflecting the rescaling of the time unit, see Figs. 6d and 6e. Realistically, the linearity between $\epsilon_{1}$ and $v_{1}$ will however break down for very small velocities, as otherwise nonmoving motors would never unbind.
While the relative magnitudes of all rates within state $C_{2}$ are unaffected by the mode of varying the velocity, the ratio of the unbinding rate $\epsilon_{2}$ and the binding rate $\pi$ is affected by this variation. As a consequence, the cargo run length now exhibits the opposite behavior as in the case above. Figure $6 f$ shows that the run length is strongly increased for decreasing single motor velocities, as the probability of having both motors bound to the filament increases strongly due to the reduction of the unbinding rate $\epsilon_{2}$. This is exactly the effect observed by $\mathrm{Xu}$ et al. ${ }^{63}$ with two kinesin motors, who found the run length of two coupled motors to increase when the single motor velocity was decreased.

In summary, a reduction of the single motor velocity has two effects on the cargo run length beyond the obvious direct linear dependence on the velocity: (i) The two-motor unbinding rate $\epsilon_{2}$ tends to decrease as strain is built up more slowly and interference effects are reduced. This decrease of the unbinding rate acts to increase the run length, which however is not sufficient to overcome the reduction from the decreasing velocity. (ii) If the decrease of the motor velocity is coupled to a reduction of the unbinding rate, as observed for changes via the ATP concentration, the unbinding rate $\epsilon_{2}$ decreases relative to the rebinding rate $\pi$ and thus the cargo spends more and more time bound via both motors. This again increases the run length, and this increase is now large enough to overcome the reduction via the decreasing velocity. Finally, we note that as already mentioned, the linearity between $v_{1}$ and $\epsilon_{1}$ will not be valid for arbitrarily small motor velocities or, if the velocity is modulated via the ATP concentration, only for a certain range of ATP concentrations, so that a real system will exhibit a combination of the two behaviors discussed here. A detailed analysis of the ATP-dependence of motor cooperation therefore requires an explicit model for the chemomechanical cycle of the motors. ${ }^{37}$

\section{CONCLUDING REMARKS}

In this article, we have reviewed and extended our previous study ${ }^{7}$ of pairs of coupled molecular motors and discussed effects of nonlinear elasticity and of the modulation of the single motor velocity (e.g. by varying the ATP concentration). We have emphasized that a large number of observations can be understood by comparing time scales for the built-up of strain forces between the motors with the time scale for spontaneous unbinding: If strain is generated slowly, motors unbind spontaneously before sufficient forces have been built up. In that case, the elastic coupling between the motors has almost no effect on the dynamics of a 
cargo pulled by a motor pair. If, however, strain is built up rapidly, the coupling leads to interference between the motors which may lead to enhanced unbinding or/and a reduced velocity. These four dynamic behaviors define the distinct transport regimes discussed in our earlier work. ${ }^{7}$ Which behavior is observed depends on two properties of the motor pair: (i) It depends on the parameters of the individual motors, i.e. on the motor type. For coupled kinesin-1 motors, we find that enhanced unbinding is the dominant effect, while for coupled myosin V motors, our model predicts a reduced velocity. Both theoretical results are in agreement with experimental observations; ${ }^{48,53}$ and (ii) it also depends on the strength and the type of the coupling as shown for example by the results of Fig. 2. These effects have not been addressed experimentally yet, but the coupling strength could be varied in engineered motor complexes by using different linkers for the two motors.

Throughout this article, we have considered the motor pair as not experiencing an external force. An obvious next step in the analysis described here is an extension to the case where the motors work against an external load force. This case is expected to involve additional time scales, for example the time over which imbalances in the loads experienced by the two motors disappear and equal load sharing is established.

\section{APPENDIX A: THEORETICAL DESCRIPTION OF SINGLE MOTORS}

In this appendix, we briefly summarize the description of single motors that we use as an ingredient for the model of coupled motors. This description is based on the measured force-dependencies of three dynamic processes: motor binding to the filament, unbinding from the filament, and stepping. For simplicity we only consider forward steps, but backward steps can also be taken into account by a straightforward extension of the model. ${ }^{7}$ The essential ingredient of our model is the force-dependence of the rates of these three processes.

The fore-dependence of the unbinding rate is described by

$$
\epsilon_{1}(F)=\epsilon_{0} \exp \left(|F| / F_{\mathrm{d}}\right),
$$

an exponential increase with the absolute value of the force $F$, as suggested by experiments with kinesin- $1^{56}$ and by theory, ${ }^{4}$ see Fig. 7c. Here, the force is scaled to the detachment force $F_{\mathrm{d}}$ which is of the order of $p N .^{56}$ The detachment force can be considered as the characteristic force that the motor can sustain for an extended period of time before unbinding. When backward steps are not modeled explicitly, the forcedependence of the stepping rate $\alpha$ is obtained from the force-velocity relation $\mathcal{V}(F)$ through $\alpha(F)=\mathcal{V}(F) / l$, where $l$ is the step length.

Typically, the velocity of a motor decreases with an opposing force until the motor stops under the stall force $F_{\mathrm{s}}$. For assisting forces, the velocity of kinesin can be taken as constant, as found experimentally for kinesin. ${ }^{11}$ To capture kinesin's behavior, we describe the force-velocity relation by a piecewise linear function,

$$
\mathcal{V}(F) \equiv\left\{\begin{array}{cc}
v_{1} & F<0 \\
v_{1}\left(1-F / F_{\mathrm{s}}\right) & 0 \leq F<F_{\mathrm{s}} \\
0 & F \geq F_{\mathrm{s}},
\end{array}\right.
$$

which involves two parameters: the stall force $F_{\mathrm{s}}$ as the characteristic force for stalling and the velocity $v_{1}$ for the force-free case, see Fig. 7b. Other functional forms of the force-velocity relation, including non-constant behavior for assisting forces can be implemented in the same way, as shown in Berger et al. ${ }^{7}$ Finally, we take the binding rate $\pi$ as independent of the force.

A few comments are in order: (i) Here we use simple expressions for the force-dependence of the unbinding rate and the velocity aimed more at capturing the core (a)

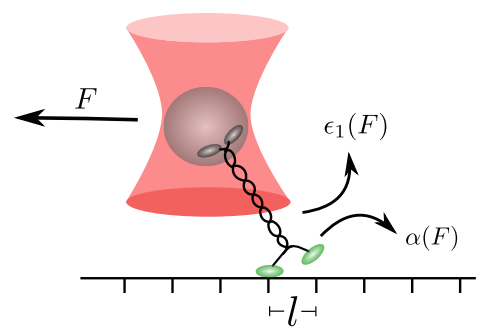

(b)

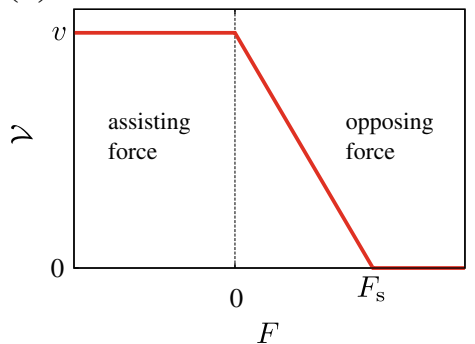

(c)

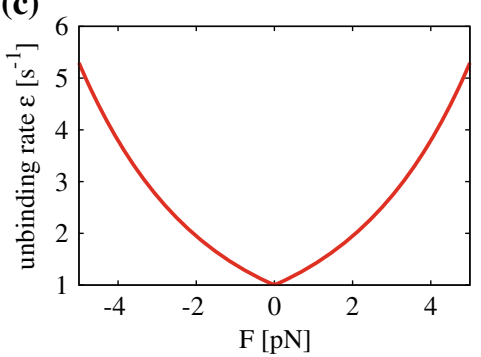

FIGURE 7. Force-dependent dynamics of a single motor: (a) Sketch of a typical single molecule experiment, in which a single kinesin is held with an optical trap which exerts the force $F$ on the motor in direction opposite to its walking direction. The motor steps forward with the force-dependent stepping rate $\alpha(F)$ and the step size $I$. Force-dependent unbinding of the motor from the filament is described by the rate $\epsilon_{1}(\boldsymbol{F})$; (b) piecewise-linear parametrization of the force-velocity relation $\mathscr{V}(\boldsymbol{F})$ from which the stepping rate is determined via $\alpha(F)=\mathscr{V}(F) / I$; (c) parametrization of the force-dependent unbinding rate of the single motor. 
properties of the observed dependencies than at a precise quantitative description. Complex, but more accurate expressions can however be incorporated into our modeling approach. Likewise, the approach can easily be adapted to molecular motors whose dynamic parameters exhibit different force-dependencies (an example would be the different force-dependence of unbinding for dynein ${ }^{43}$ ). (ii) In this description of the motor the internal chemomechanical states of motor proteins ${ }^{9,46}$ are not described explicitly. All effects due to the different mechanochemical states must be accounted for implicitly via the rates $\alpha, \epsilon_{1}$ and $\pi$. Effects of the chemomechanical cycles will be studied elsewhere. ${ }^{37}$ (iii) In total, this description of a single motor depends on six parameters. Their values for kinesin-1 are summarized in Table 1. The number of parameters will in general increase if more complex descriptions of the force-dependencies are used.

\section{APPENDIX B: THEORETICAL DESCRIPTION OF THE BINDING PROCESS}

In this appendix, we provide a justification for our description of the binding process by a constant binding rate. Other more complicated approaches have been discussed in several studies. ${ }^{19,20,35,48}$ Our approach is based on the observation that binding and unbinding proceed along different pathways: whereas unbinding dominantly occurs from strained configurations (all states $(2, i)$ with $i>0)$, motors mostly bind in a relaxed configuration, i. e. to state $(2,0)$. Therefore, we describe binding with a single rate that is independent of the force.

In the following, we expand on this picture of the binding process. If the cargo is bound only by one motor to the filament, the other motor is in a relaxed, strain-free configuration. The unbound motor searches diffusively for a binding site. In the case of strong coupling, it is rather rare that thermal fluctuations push the motor to a binding site that requires binding under strain. The dominant binding configuration is the relaxed configuration $(2,0)$, as assumed in the main text. For small stiffness $\kappa$, it is more likely that the motor also binds to different states $(2, i)$ in which the motor is stretched. However, since, in this case, the coupling strength $\kappa$ is small, the overall induced strain force that influences unbinding and stepping is also small.

To estimate the effect of binding under strain for intermediate coupling strength, we assume that the probability distribution of the unbound motor to bind to state $(2, i)$ is given by the Boltzmann distribution $P_{i}^{\text {init }} \equiv \mathcal{N}^{-1} \mathrm{e}^{-\kappa(l i)^{2} / 2 k_{\mathrm{B}} T} \quad$ with normalization $\mathcal{N}=$ $\sum_{i} \mathrm{e}^{-\kappa(i i)^{2} / 2 k_{\mathrm{B}} T}$. Then, the average elastic strain force under which binding of the second motor occurs, is given as $\langle F\rangle^{\text {init }} \equiv \sum_{i} F_{i} P_{i}^{\text {init }}$, with the elastic strain force $F_{i}=\frac{1}{2} \kappa l i$. We calculated this quantity. For the kinesin parameter given in Table 1 and $k_{\mathrm{B}} T \simeq 4.1 \mathrm{pN} \mathrm{nm}$, we found that its maximal value is very small, $0.15 \mathrm{pN}$, at an elastic coupling strength of about $0.17 \mathrm{pN} / \mathrm{nm}$. As a consequence, the overall effect of binding under strain is negligible and the restriction that the motor rebinds only in its relaxed state provides a very good approximation.

\section{APPENDIX C: DETAILED CALCULATION OF $\epsilon_{2}$ AND $V_{2}$}

In the following, we describe the method we use to calculate the effective unbinding rate $\epsilon_{2}$ and the velocity $v_{2}$ of two motors bound to the filament simultaneously. ${ }^{5,7}$ We consider the discrete network of states depicted in the lower row of Fig. 1. This network describes the strain force-dependent dynamics of the motor pair in terms of their stepping and unbinding. We assume that the two motors start working together in the state $(2,0)$ in which both motor linkers are relaxed. The state $(2, N)$ corresponds to a force, which is equal or large the single motor stall force, such that the motors are not able to step anymore. The effective unbinding rate is then determined by the inverse mean first passage time to reach state $\left(C_{1}\right)$ provided that the process has started in state $(2,0)$. The velocity $v_{2}$ is determined by the average stepping rate times the displacement of the cargo per motor step. Technically speaking, we consider a Markov process on the network shown in Fig. 1 with an absorbing state $\left(C_{1}\right)$. In general, average quantities such as the mean time to absorption or the average stepping rate can be obtained from the time-dependent probability distribution for the network. However, a more intuitive and elegant way to obtain these quantities has been proposed by Hill. ${ }^{28,29}$ The basic idea is to use the ensemble average instead of the time average. The ensemble average is determined using a time-independent probability distribution that is obtained from a modified network without the absorbing state: As soon as the process reaches the absorbing state, it is re-started at the initial state. A network for such dynamics is obtained by eliminating the absorbing state and redirecting all transitions directed towards the absorbing state to the initial state. In this way the original network shown in Fig. 1 is closed to the modified network shown in Fig. 8. The probability distribution $P_{2, i}$ for the closed network is determined from the solution of the steady state master equation, 


$$
\begin{gathered}
\partial_{t} P_{2,0}=-\left[\omega_{s}(2,0)+\omega_{\text {off }}(2,0)\right] P_{2,0}+\omega_{r}(2,1) P_{2,1} \\
+\sum_{j=0}^{N} \omega_{\text {off }}(2, j) P_{2, j} \\
\partial_{t} P_{2, i}=\omega_{s}(2, i-1) P_{2, i-1}-\left[\omega_{s}(2, i)+\omega_{r}(2, i)\right. \\
\left.+\omega_{\text {off }}(2, i)\right] P_{2, i}+\omega_{r}(2, i+1) P_{2, i+1} \\
\quad \text { for } 0<i<N \\
\partial_{t} P_{2, N}=\omega_{s}(2, N-1) P_{2, N-1}-\left[\omega_{r}(2, N)\right. \\
\left.\quad+\omega_{\text {off }}(2, N)\right] P_{2, N} .
\end{gathered}
$$

Here $P_{2, i}$ is the probability of being in state $(2, i)$ before absorption. Together with the normalization condition, this set of equations can be solved with a backward substitution, since $P_{2, N}$ only depends on $P_{2, N-1}$. Now, the inverse mean first passage time is the rate of being absorbed, which is given by probability current into the absorbing state,

$$
\epsilon_{2}=\sum_{i=0}^{N} \omega_{\mathrm{off}}(2, i) P_{2, i}
$$

Averaging the stepping rates of both motors, we obtain the velocity

$$
v_{2}=\frac{l}{2} \sum_{i=0}^{N}\left[\alpha\left(F_{i}\right)+\alpha\left(-F_{i}\right)\right] P_{2, i}
$$

\section{APPENDIX D: THEORETICAL DESCRIPTION OF CABLE-LIKE LINKERS}

To be able to describe cable-like linkers within our detailed model for two coupled motors, we have to extend the state space of Fig. 1. We add $N^{-}$states with a negative index, which are associated with the compression mode, i.e. $F_{i}=0$ for all these states. The choice of a negative index ensures that the state in which the motors are separated by one step length still corresponds to state $(2,1)$ as before. The number $N^{-}$of these states depends on the rest length $l_{0}$. For example, if $l_{0}=0$, there is no compression mode and therefore $N^{-}=0$ and we recover the case of a linear spring. For convenience, we choose the rest length $l_{0}$ to be a multiple of the step size $l$ and therefore $N^{-}=2 l_{0} / l$. Otherwise an appropriate rounding function has to be introduced. We illustrate the system in Fig. 9, in which the shaded gray areas are associated with the compression modes. Because of the assumed geometry, we do not distinguish whether motors walk towards each other or away from each other, compare Fig. 1. Therefore, we assign to every state $\left(2,-N^{-}+1\right) \ldots(N)$ two configurations that correspond to the two columns in Fig. 9. The only state with a unique configuration is the state $\left(N^{-}\right)$, in which the effective distance is zero. Accordingly, we adjust the rates for stretching, relaxation and unbinding:

$$
\begin{gathered}
\omega_{s}(2, i)=\left\{\begin{array}{cc}
2 \alpha(0) & i=N^{-} \\
\alpha(0) & N^{-}<i \leq 0 \\
\alpha\left(F_{i}\right) & 0<i<N,
\end{array}\right. \\
\omega_{r}(2, i)=\left\{\begin{array}{cc}
\alpha(0) & N^{-}<i \leq 0 \\
\alpha\left(-F_{i}\right) & 0<i \leq N,
\end{array}\right. \\
\omega_{\text {off }}(2, i)=\left\{\begin{array}{cc}
2 \epsilon_{1}(0) & N^{-} \leq i \leq 0 \\
\epsilon_{1}\left(F_{i}\right)+\epsilon_{1}\left(-F_{i}\right) & 0<i \leq N .
\end{array}\right.
\end{gathered}
$$

We note that for $N^{-}=0$, the case that corresponds to a simple linear spring, we recover the rates specified in Eqs. (5)-(7).

In the extended model, there are $N^{-}+1$ force-free states, in which the linkers are relaxed. We consider all of these states as possible initial states and therefore assume that when on motor is active, the other motor binds with equal probability $w=1 /\left(N^{-}+1\right)$ into any of these $N^{-}+1$ force-free states.

With these extensions of the model, the unbinding rate $\epsilon_{2}$ and the velocity $v_{2}$ are then determined in the same way as for the original model described in the main text.

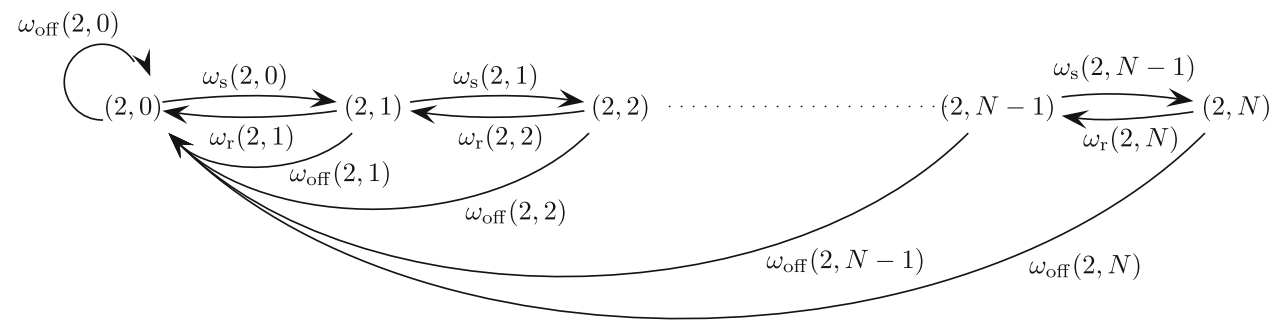

FIGURE 8. Closed version of the network of network in the lower row shown in Fig. 1 obtained by redirecting all arrows that lead into the absorbing state back into the initial starting state $(2,0)$. 


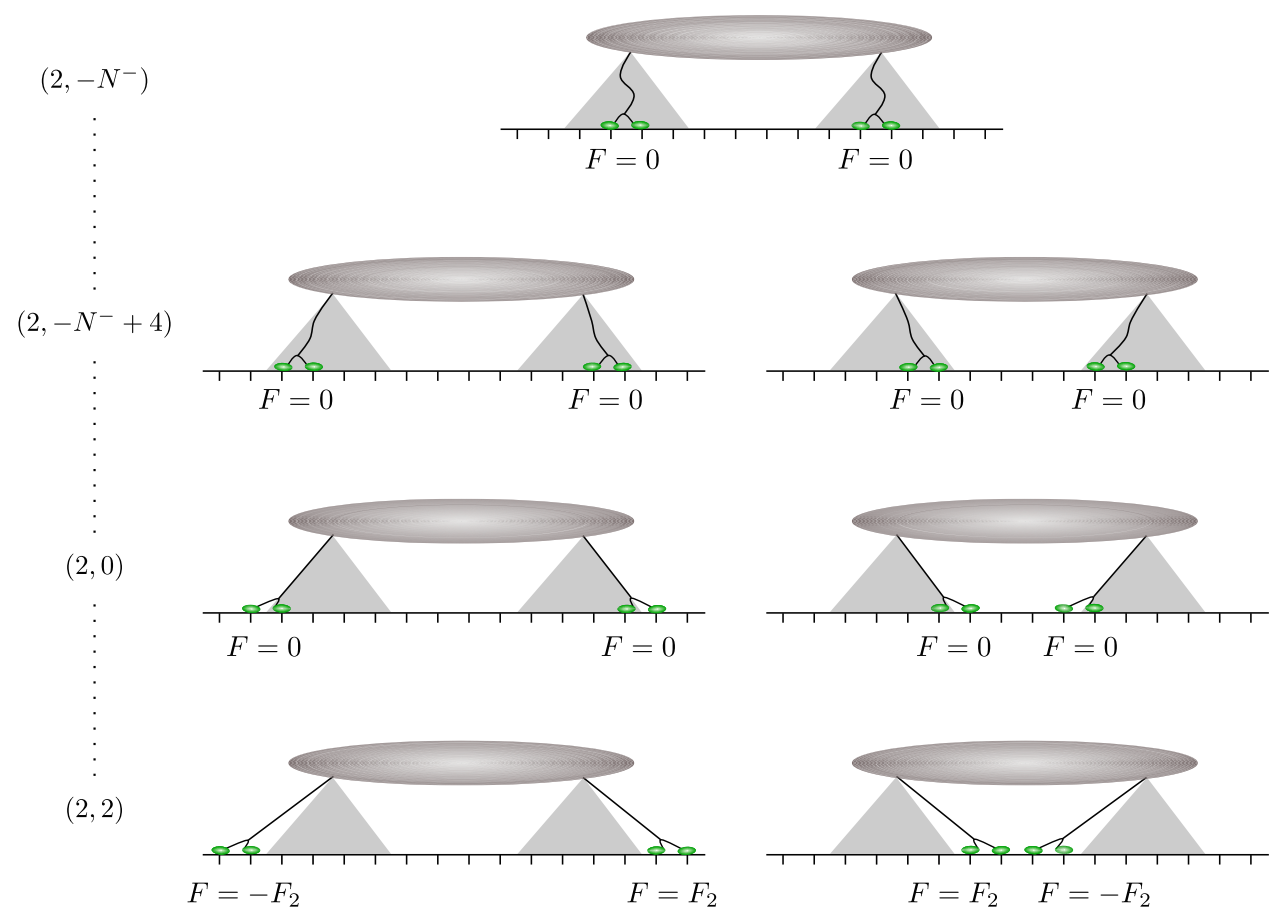

FIGURE 9. State space and the corresponding motor configurations. Because of the geometry assumed, all states except for state $\left(2,-N^{-}\right)$have two motor configurations. The areas shaded in gray are associated with the compression mode of the linkers. When the motors are in this area, no force is built up because their linkers are relaxed.

\section{ACKNOWLEDGMENTS}

The authors thank Melanie Müller and Veronika Bierbaum for stimulating discussions.

\section{REFERENCES}

${ }^{1}$ Ali, M. Y., G. G. Kennedy, D. Safer, K. M. Trybus, H. Lee Sweeney, and D. M. Warshaw. Myosin Va and myosin VI coordinate their steps while engaged in an in vitro tug of war during cargo transport. Proc. Natl. Acad. Sci. U.S.A. 108:E535, 2011.

${ }^{2}$ Atzberger, P. J., and C. S. Peskin. A brownian dynamics model of kinesin in three dimensions incorporating the force-extension profile of the coiled-coil cargo tether. Bull. Math. Biol. 68:131, 2006.

${ }^{3}$ Beeg, J., S. Klumpp, R. Dimova, R. S. Graciá, E. Unger, and R. Lipowsky. Transport of beads by several kinesin motors. Biophys. J. 94:532, 2008.

${ }^{4}$ Bell, G. I. Models for the specific adhesion of cells to cells. Science 200:618, 1978.

${ }^{5}$ Berger, F. (2012) Ph.D Thesis: Different Modes of Cooperative Transport by molecular motors. University of Potsdam. ${ }^{6}$ Berger, F., C. Keller, M. J. I. Müller, S. Klumpp, and R. Lipowsky. Co-operative transport by molecular motors. Biochem. Soc. Trans. 39:1211, 2011.

${ }^{7}$ Berger, F., C. Keller, S. Klumpp, and R. Lipowsky. Distinct transport regimes of two elastically coupled molecular motors. Phys. Rev. Lett. 108:208101, 2012.

${ }^{8}$ Bieling, P., I. A. Telley, J. Piehler, and T. Surrey. Processive kinesins require loose mechanical coupling for efficient collective motility. EMBO Rep. 9:1121, 2008.
${ }^{9}$ Bierbaum, V., and R. Lipowsky. Chemomechanical coupling and motor cycles of myosin V. Biophys. J. 100:1747, 2011.

${ }^{10}$ Block, S. M., L. S. B. Goldstein, and B. J. Schnapp. Bead movement by single kinesin molecules studied with optical tweezers. Nature 348:348, 1990.

${ }^{11}$ Block, S. M., C. L. Asbury, J. W. Shaevitz, and M. J. Lang. Probing the kinesin reaction cycle with a $2 \mathrm{~d}$ optical force clamp. Proc. Natl. Acad. Sci. U.S.A. 100:2351, 2003.

${ }^{12}$ Böhm, K. J., R. Stracke, P. Mühlig, and E. Unger. Motor protein-driven unidirectional transport of micrometer-sized cargoes across isopolar microtubule arrays. Nanotechnology 12:238, 2001.

${ }^{13}$ Bouzat, S., and F. Falo. The influence of direct motormotor interaction in models for cargo transport by a single team of motors. Phys. Biol. 7:046009, 2010.

${ }^{14}$ Campás, O., Y. Kafri, K. B. Zeldovich, J. Casademunt, and J.-F. Joanny. Collective dynamics of interacting molecular motors. Phys. Rev. Lett. 97:038101, 2006.

${ }^{15}$ Constantinou, P. E., and M. R. Diehl. The mechanochemistry of integrated motor protein complexes. J. Biomech. 43:31, 2010.

${ }^{16}$ Coppin, C., D. Pierce, L. Hsu, and R.Vale. The load dependence of kinesin's mechanical cycle. Proc. Natl. Acad. Sci. U.S.A. 94:8539, 1997.

${ }^{17}$ Coy, D. L., M. Wagenbach, and J. Howard. Kinesin takes one 8-nm step for each ATP that it hydrolyzes. J. Biol. Chem. 274:3667, 1999.

${ }^{18}$ Dixit, R., J. L. Ross, Y. E. Goldman, and E. L. F. Holzbaur. Differential regulation of dynein and kinesin motor proteins by ta. Science 319:1086, 2008.

${ }^{19}$ Driver, J. W., A. R. Rogers, D. K. Jamison, R. K. Das, A. B. Kolomeisky, and M. R. Diehl. Coupling between motor proteins determines dynamic behaviors of motor protein assemblies. Phys. Chem. Chem. Phys. 12:10398, 2010. 
${ }^{20}$ Driver, J. W., D. K. Jamison, K. Uppulury, A. R. Rogers, A. B. Kolomeisky, and M. R. Diehl. Productive cooperation among processive motors depends inversely on their mechanochemical efficiency. Biophys. J. 101:386, 2011.

${ }^{21}$ Erickson, R. P., Z. Jia, S. P. Gross, and C. C. Yu. How molecular motors are arranged on a cargo is important for vesicular transport. PLoS Comput. Biol. 7:e1002032, 2011.

${ }^{22}$ Fisher, M. E., and A. B. Kolomeisky. Simple mechanochemistry describes the dynamics of kinesin molecules. Proc. Natl. Acad. Sci. U.S.A. 98:7748, 2001.

${ }^{23}$ Gagliano, J., M. Walb, B. Blaker, J. C. Macosko, and G.Holzwarth. Kinesin velocity increases with the number of motors pulling against viscoelastic drag. Eur. Biophys. J. 39:801, 2010.

${ }^{24}$ Gross, S. P. Hither and yon: a review of bi-directional microtubule-based transport. Phys. Biol. 1:R1, 2004.

${ }^{25}$ Gross, S. P., M. Vershinin, and G. T. Shubeita. Cargo transport: two motors are sometimes better than one. Curr. Biol. 17:R478, 2007.

${ }^{26}$ Hess, H. Engineering applications of biomolecular motors. Annu. Rev. Biomed. Eng. 13:429, 2011.

${ }^{27}$ van den Heuvel, M. G. L., and C. Dekker. Motor proteins at work for nanotechnology. Science 317:333, 2007.

${ }^{28}$ Hill, T. L. Interrelations between random walks on diagrams (graphs) with and without cycles. Proc. Natl. Acad. Sci. U.S.A. 85:2879, 1988.

${ }^{29}$ Hill, T. L. Number of visits to a state in a random walk, before absorption, and related topics. Proc. Natl. Acad. Sci. U.S.A. 85:4577, 1988.

${ }^{30}$ Holzbaur, E. L., and Y. E. Goldman. Coordination of molecular motors: from in vitro assays to intracellular dynamics. Curr. Opin. Cell Biol. 22:4, 2010.

${ }^{31}$ Howard, J. Mechanics of Motor Proteins and the Cytoskeleton. Sunderland, MA: Sinauer Associates, 2001.

${ }^{32}$ Huxley, H. E., and J. Hanson. Changes in the cross-striations of muscle during contraction and stretch and their structural interpretation. Nature 173:973, 1954.

${ }^{33}$ Huxley, A. F., and R. Niedergerke.Structural changes in muscle during contraction: interference microscopy of living muscle fibres. Nature 173:971, 1954.

${ }^{34}$ Jamison, D. K., J. W. Driver, A. R. Rogers, P. E. Constantinou, and M. R. Diehl. Two kinesins transport cargo primarily via the action of one motor: Implications for intracellular transport. Biophys. J. 99:2967, 2010.

${ }^{35}$ Jamison, D. K., J. W. Driver, and M. R. Diehl. Cooperative responses of multiple kinesins to variable and constant loads. J. Biol. Chem. 287:3357, 2012.

${ }^{36}$ Keller, C. Diploma Thesis: Coupled molecular motors. Humboldt University of Berlin, 2009.

${ }^{37}$ Keller, C., F. Berger, S. Liepelt, and R. Lipowsky. Network complexity and parametric simplicity for cargo transport by two molecular motors, 2012 (submitted).

${ }^{38}$ Klumpp, S., and R. Lipowsky. Cooperative cargo transport by several molecular motors. Proc. Natl. Acad. Sci. U.S.A. 102:17284, 2005.

${ }^{39}$ Korn, C. B., S. Klumpp, R. Lipowsky, and U. S. Schwarz. Stochastic simulations of cargo transport by processive molecular motors. J. Chem. Phys. 131:245107, 2009.

${ }^{40}$ Korten, T., and A. Månsson. Towards the application of cytoskeletal motor proteins in molecular detection and diagnostic devices. Diez S. Curr. Opin. Biotech. 21:477, 2010.

${ }^{41}$ Kunwar, A., and A. Mogilner. Robust transport by multiple motors with nonlinear force-velocity relations and stochastic load sharing. Phys. Biol. 7:016012, 2010.
${ }^{42}$ Kunwar, A., M. Vershinin, J. Xu, and S. P. Gross. Stepping, strain gating, and an unexpected force-velocity curve for multiple-motor-based transport. Curr. Biol. 18:1173, 2008.

${ }^{43}$ Kunwar, A., S. K. Tripathy, J. Xu, M. K. Mattson, P. Anand, R. Sigua, M. Vershinin, R. J. McKenney, C. C. Yu, A. Mogilner, et al. Mechanical stochastic tug-of-war models cannot explain bidirectional lipid-droplet transport. Proc. Natl. Acad. Sci. U.S.A. 108:18960, 2011.

${ }^{44}$ Leduc, C., O. Camp'as, K. B. Zeldovich, A. Roux, P. Jolimaitre, L. Bourel-Bonnet, B. Goud, J. F. Joanny, P. Bassereau, and J. Prost. Cooperative extraction of membrane nanotubes by molecular motors. Proc. Natl. Acad. Sci. U.S.A. 101:17096, 2004.

${ }^{45}$ Leduc, C., F. Ruhnow, J. Howard, and S. Diez. Detection of fractional steps in cargo movement by the collective operation of kinesin-1 motors. Proc. Natl. Acad. Sci. U.S.A. 104:10847, 2007.

${ }^{46}$ Liepelt, S., and R. Lipowsky. Kinesin's network of chemomechanical motor cycles. Phys. Rev. Lett. 98:258102, 2007.

${ }^{47}$ Lipowsky, R., and S. Klumpp. 'Life is motion': multiscale motility of molecular motors. Physica A 352:53, 2005.

${ }^{48} \mathrm{Lu}, \mathrm{H}$, A. K. Efremov, C. S. Bookwalter, E. B. Krementsova, J. W. Driver, K. M. Trybus, and M. R. Diehl. Collective dynamics of elastically-coupled myosin V motors. J. Biol. Chem. 287:27753, 2012.

${ }^{49}$ Mallik, R., D. Petrov, S. A. Lex, S. King, and S. Gross. Building complexity: an in vitro study of cytoplasmic dynein with in vivo implications. Curr. Biol. 15:2075, 2005.

${ }^{50}$ Müller, M. J. I., S. Klumpp, and R. Lipowsky. Tug-of-war as a cooperative mechanism for bidirectional cargo transport by molecular motors. Proc. Natl. Acad. Sci. U.S.A. 105:4609, 2008.

${ }^{51}$ Müller, M. J. I., S. Klumpp, and R. Lipowsky. Motility states of molecular motors engaged in a stochastic tug-ofwar. J. Stat. Phys. 133:1059, 2008.

${ }^{52}$ Müller, M. J. I., S. Klumpp, and R. Lipowsky. Bidirectional transport by molecular motors: Enhanced processivity and response to external forces. Biophys. J. 98:2610. 2010.

${ }^{53}$ Rogers, A. R., J. W. Driver, P. E. Constantinou, D. K. Jamison, and M. R. Diehl. Negative interference dominates collective transport of kinesin motors in the absence of load. Phys. Chem. Chem. Phys. 11:4882, 2009.

${ }^{54}$ Schief, W. R., R. H. Clark, A. H. Crevenna, and J. Howard. Inhibition of kinesin motility by ADP and phosphate supports a hand-over-hand mechanism. Proc. Natl. Acad. Sci. U.S.A. 101:1183, 2004.

${ }^{55}$ Schliwa M., and G. Woehlke, Molecular motors. Nature 422:759, 2003.

${ }^{56}$ Schnitzer, M., K. Visscher, and S. Block. Force production by single kinesin motors. Nat. Cell. Biol. 2:718, 2000.

${ }^{57}$ Uppulury, K., A. K. Efremov, J. W. Driver, D. K. Jamison, M. R. Diehl, and A. B. Kolomeisky. Motor proteindriven unidirectional transport of micrometer-sized cargoes across isopolar microtubule arrays. J. Phys. Chem. B 116:8846, 2012.

${ }^{58}$ Veigel, C., and C. F. Schmidt. Moving into the cell: singlemolecule studies of molecular motors in complex environments. Nat. Rev. Mol. Cell Biol. 12:163, 2011.

${ }^{59}$ Verbrugge, S., S. M. J. L. van den Wildenberg, and E. J. G. Peterman. Novel ways to determine kinesin-1's run length and randomness using fluorescence microscope. Biophys. J. 97:2287, 2009.

${ }^{60}$ Vershinin, M., B. C. Carter, D. S. Razafsky, S. J. King, and S. P.Gross. Multiple-motor based transport and its regulation by tau. Proc. Natl. Acad. Sci. U.S.A. 104:87, 2007. 
${ }^{61}$ Wang, Z., and M. Li. Force-velocity relations for multiplemolecular-motor transport. Phys. Rev. E 80:041923, 2009.

${ }^{62}$ Welte, M. A. Bidirectional transport along microtubules. Curr. Biol. 14:R525, 2004.
${ }^{63}$ Xu, J., Z. Shu, S. J. King, and S. P.Gross. Tuning multiple motor travel via single motor velocity. Traffic 13:1198, 2012.

${ }^{64}$ Zhang, Y. Cargo transport by several motors. Phys. Rev. E 83:011909, 2011. 